\title{
A Study Involving the Completion of a Quasi-2-Normed Space
}

\author{
Mehmet Kir ${ }^{1}$ and Mehmet Acikgoz ${ }^{2}$ \\ ${ }^{1}$ Department of Mathematics, Faculty of Science, Atatürk University, 25000 Erzurum, Turkey \\ ${ }^{2}$ Department of Mathematics, Faculty of Science and Arts, University of Gaziantep, 27310 Gaziantep, Turkey
}

Correspondence should be addressed to Mehmet Kır; mehmet_040465@yahoo.com

Received 9 August 2012; Accepted 29 January 2013

Academic Editor: Chuanxi Qian

Copyright (C) 2013 M. Kır and M. Acikgoz. This is an open access article distributed under the Creative Commons Attribution License, which permits unrestricted use, distribution, and reproduction in any medium, provided the original work is properly cited.

The fundamental aim of this paper is to introduce and investigate a new property of a quasi-2-normed space based on a question given by Park (2006) for the completion quasi-2-normed space. Finally, we also find an answer for Park's question.

\section{Introduction, Definitions, and Notations}

In 1928, Menger introduced the notion called n-metrics (or generalized metric) [1]. But many mathematicians had not paid attentions to Menger's theory about generalized metrics. But several mathematicians, A. Wald, L. M. Blumenthal, W. A. Wilson, and so forth, have developed Menger's idea.

In 1965, Gähler limits Menger's considerations to $n=2$. Gähler's study is more complete in view of the fact that he develops the topological properties of the spaces in question. Gähler also proves that if the space is a linear normed space, then it is possible to define 2-norm [2].

Since 1963, S. Gähler, Y. J. Cho, R. W. Frees, C. R. Diminnie, R. E. Ehret, K. Iséki, A. White, and many others have studied on 2-normed spaces and 2-metric spaces. It is well known that $\mathbb{R}$ is complete but $\mathbb{Q}$ is not complete. Since $\mathbb{Q}$ is dense in $\mathbb{R}$, it is the said that $R$ is completion of $\mathbb{Q}$. It is very important that an incomplete space can be completed in similar sense. Complete spaces, in other words Banach spaces, play quite important role in many branches of mathematics and its applications. Many mathematicians showed the existence of the completion of normed spaces (for more information, see [3-5]). We will also show the completion of quasi-2-normed spaces via similar sense.

Definition 1. Let $X$ be a real linear space with $\operatorname{dim} \geq 2$, and let $\|\cdot, \cdot\|: X^{2} \rightarrow[0, \infty)$, be a function. Then, let $(X,\|\cdot, \cdot\|)$ is called linear 2-normed spaces if

$\left(2 N_{1}\right)\|x, y\|=0 \Leftrightarrow x$ and $y$ are linearly dependent,

$\left(2 N_{2}\right)\|x, y\|=\|y, x\|$ $\left(2 N_{3}\right)\|\alpha x, y\|=|\alpha|\|x, y\|$,

$\left(2 N_{4}\right)\|x+y, z\| \leq\|x, z\|+\|y, z\|$,

for all $\alpha \in \mathbb{R}$ and all $x, y, z \in X$.

Example 2. Let $E_{3}$ denotes the Euclidean vector three spaces. Let $x=a i+b j+c k$ and $y=d i+e j+f k$ define

$$
\begin{aligned}
\|x, y\| & =|x \times y|=\operatorname{abs}\left|\begin{array}{lll}
i & j & k \\
a & b & c \\
d & e & f
\end{array}\right| \\
& =\left|(b f-c e)^{2} i+(c d-a f)^{2} j+(a e-d b)^{2} k\right|^{1 / 2} .
\end{aligned}
$$

Then, $\left(E_{3},\|\cdot, \cdot\|\right)$ is a 2-normed space, and this space is complete (for more information, see [5]).

Also,

(1) in addition to $\left(2 N_{1}\right),\left(2 N_{2}\right)$, and $\left(2 N_{3}\right)$, if there is a constant $K \geq 1$ such that

$\left(2 N_{4}^{*}\right)\|x+y, z\| \leq K(\|x, z\|+\|y, z\|)$, for all $x, y, z \in$ $X$ is called a quasi-2-normed space;

(2) a 2-norm $\|\cdot, \cdot\|$ defined on a linear space $X$ is said to be uniformly continuous in both variables, if for any $\varepsilon>0$, there exists a neighbourhood $U_{\varepsilon}$ of 0 such that $\left|\|a, b\|-\left\|a^{\prime}, b^{\prime}\right\|\right|<\varepsilon$, whenever $a-a^{\prime}$ and $b-b^{\prime}$ are in $U_{\varepsilon}$, which is independent of the choice of $a, a^{\prime}, b, b^{\prime}$;

(3) a pseudo-2-norm is defined to be real-valued function having all the properties of 2 -norm $\|\cdot, \cdot\|$ except 
the condition that $\|a, b\|=0$ implies the linear dependence of $a$ and $b$ (for details, see [3]).

Example 3. Let $X$ be a linear space with $\operatorname{dim} X \geq 2$, and let $\|\cdot, \cdot\|$ be 2 -norm on $X$.

$$
\|x, y\|_{q}=2\|x, y\|
$$

is quasi-2-norm on $X$, and $\left(X,\|\cdot, \cdot\|_{q}\right)$ is a quasi-2-normed space.

Solution 1. By using conditions $\left(2 N_{1}\right),\left(2 N_{2}\right)$, and $\left(2 N_{3}\right)$ in 2 -normed spaces, however, using $\left(2 N_{4}^{*}\right)$, we show that

$\left(2 N_{1}\right)\|x, y\|_{q}=0$ if and only if $2\|x, y\|=0$, namely, $x$ and $y$ are linearly dependent:

It is easy to see for $\left(2 N_{2}\right)$, that is, $\|x, y\|_{q}=\|y, x\|_{q}$, using the property of $\left(2 N_{3}\right)$, we readily see the following applications: for all $\alpha \in \mathbb{R}$,

$$
\begin{aligned}
\|\alpha x, y\|_{q} & =2\|\alpha x, y\|=|\alpha|(2\|x, y\|) \\
& =|\alpha|\|x, y\|_{q},
\end{aligned}
$$

we are now ready to prove the property of $\left(2 N_{4}^{*}\right)$, that is, for all $x, y, z \in X$

$$
\begin{aligned}
\|x+y, z\|_{q} & =2\|x+y, z\| \\
& \leq 2(\|x, z\|+\|y, z\|) \\
& =\|x, z\|_{q}+\|y, z\|_{q},
\end{aligned}
$$

so, we complete the solution of Example 3.

Theorem 4. Let $X$ be a linear space with $\operatorname{dim} X \geq 2$, and let $\|\cdot, \cdot\|$ be 2 -norm on $X$, for constant $a, b \in \mathbb{R}$ which are $a \geq 1 / 2$ and $b \geq 1 / 2$. There exists $\|x, y\|_{q}$ quasi-2-norm on $X$ defined as

$$
\|x, y\|_{q}=a\|x, y\|+b\|x, y\|
$$

Proof. It is evident to show conditions $\left(2 N_{1}\right),\left(2 N_{2}\right)$, and $\left(2 N_{3}\right)$; therefore, It is sufficient to prove the condition of $\left(2 N_{4}^{*}\right)$ as follows: For all $x, y, z \in X$

$$
\begin{aligned}
\|x+y, z\|_{q} & =a\|x+y, z\|+b\|x+y, z\| \\
& \leq a(\|x, z\|+\|y, z\|)+b(\|x, z\|+\|y, z\|) \\
& =(a+b)\|x, z\|+(a+b)\|y, z\| \\
& =K(\|x, z\|+\|y, z\|),
\end{aligned}
$$

since there exists a constant $K \geq 1$, namely, by substituting $K:=a+b$, we show that $\|\cdot, \cdot\|_{q}$ is a quasi-2-norm on $X$.
Definition 5. Let $(X,\|\cdot, \cdot\|)$ be a quasi-2-normed space.

(a) A sequence $\left\{x_{n}\right\}$ is a Cauchy sequence in a linear quasi-2-normed space $(X,\|\cdot, \cdot\|)$ if and only if $\lim _{n, m \rightarrow \infty}\left\|x_{n}-x_{m}, z\right\|=0$, for every $z$ in $X$.

(b) A sequence $\left\{x_{n}\right\}$ in $X$ is called a convergent sequence if there is an $x \in X$ such that $\lim _{n, m \rightarrow \infty}\left\|x_{n}-x, z\right\|=0$, for every $z$ in $X$.

(c) A quasi-2-normed space in which every Cauchy sequence converges is called complete.

Definition 6. Let $\left(X,\|\cdot, \cdot\|_{X}\right)$ and $\left(Y,\|\cdot, \cdot\|_{Y}\right)$ be quasi-2normed spaces.

(a') A mapping $T: X \rightarrow Y$ is said to be isometric or isometry, if for all $x, y \in X$,

$$
\|T x, T y\|_{Y}=\|x, y\|_{X} .
$$

$\left(b^{\prime}\right)$ The space $X$ is said to be isometry with the space $Y$ if there exists a bijective isometry of $X$ onto $Y$. The spaces $X$ and $Y$ are called isometric spaces.

Theorem 7. If a sequence $\left\{x_{n}\right\}$ is a Cauchy sequence in a linear 2 -normed space $(X,\|\cdot, \cdot\|)$, then $\lim _{n \rightarrow \infty}\left\|x_{n}, z\right\|$ exists, for every $z$ in $X$ (for proof, see [3]).

Theorem 8. If $X$ is a linear space having a uniformly continuous 2-norm $\|\cdot, \cdot\|$ defined on it, then for any two Cauchy sequences $\left\{x_{n}\right\}$ and $\left\{y_{n}\right\}$ in $X, \lim _{n \rightarrow \infty}\left\|x_{n}, y_{n}\right\|$ (for proof, see [3]).

Definition 9. The two Cauchy sequences $\left\{x_{n}\right\}$ and $\left\{y_{n}\right\}$ in a linear 2 -normed space $(X,\|\cdot, \cdot\|)$ are said to be equivalent, denoted by $\left\{x_{n}\right\} \sim\left\{y_{n}\right\}$, if for every neighbourhood $U$ of 0 , there is an integer $N(U)$ such that $n \geq N(U)$ implies that $x_{n}-y_{n} \in U$, compare [3].

\section{The Completion of a Quasi-2-Normed Space}

In [4], Park introduced quasi-2-normed spaces and gave some results on $p$-normed spaces. Also, he introduced a question which was "Construct a completion of a quasi-2norm." In this section, we give an answer to this question.

Theorem 10. $\left\{x_{n}\right\}$ is equivalent to $\left\{a_{n}\right\}$ in a linear 2-normed space $(X,\|.,\|$.$) if and only if$

$$
\lim _{n \rightarrow \infty}\left\|x_{n}-a_{n}, z\right\|=0
$$

for every $z$ in $X$ (for proof, see [3]).

Theorem 11. The relation $\sim$ on the set of the Cauchy sequences in $X$ is an equivalence relation on $X$.

Proof. It is clear that $\left\{x_{n}\right\} \sim\left\{y_{n}\right\}$ (reflexivity) and $\left\{y_{n}\right\} \sim\left\{x_{n}\right\}$ when $\left\{x_{n}\right\} \sim\left\{y_{n}\right\}$ (symmetry). 


$$
\begin{aligned}
& \text { Let }\left\{x_{n}\right\} \sim\left\{y_{n}\right\} \text { and }\left\{y_{n}\right\} \sim\left\{z_{n}\right\}, z \in X, \\
& \begin{aligned}
\lim _{n \rightarrow \infty}\left\|x_{n}-z_{n}, z\right\|= & \lim _{n \rightarrow \infty}\left\|x_{n}-y_{n}+y_{n}-z_{n}, z\right\| \\
\leq & \lim _{n \rightarrow \infty} K\left(\left\|x_{n}-y_{n}, z\right\|\right. \\
& \left.\quad+\left\|y_{n}-z_{n}, z\right\|\right), \quad K \geq 1 \\
\leq & K \lim _{n \rightarrow \infty}\left\|x_{n}-y_{n}, z\right\|+\left\|y_{n}-z_{n}, z\right\| \\
= & K(0+0)=0 .
\end{aligned}
\end{aligned}
$$

Then, $\left\{x_{n}\right\} \sim\left\{z_{n}\right\}$ (transitivity). So, $\sim$ is an equivalence relation on $X$.

Theorem 12. If $\left\{a_{n}\right\}$ and $\left\{b_{n}\right\}$ are equivalent to $\left\{x_{n}\right\}$ and $\left\{y_{n}\right\}$ in a linear 2-normed space $(X,\|\cdot, \cdot\|)$, respectively, then $\left\{a_{n}+b_{n}\right\}$ is equivalent to $\left\{x_{n}+y_{n}\right\}$ and $\left\{\alpha a_{n}\right\}$ is equivalent to $\left\{\alpha x_{n}\right\}$ (for proof, see [3]).

Denote by $\widehat{X}$ is the set of all equivalence classes of the Cauchy sequences in $X$. Let $\hat{x}, \hat{y}, \widehat{z}$, and so forth denote the elements of $\widehat{X}$. Define an addition and a scalar multiplication on $\widehat{X}$ as follows:

(i) $\hat{x}+\hat{y}$ : the set of sequences equivalent to $\left\{x_{n}+y_{n}\right\}$, where $\left\{x_{n}\right\}$ is in $\hat{x}$ and $\left\{y_{n}\right\}$ in $\hat{y}$,

(ii) $\alpha \widehat{x}$ : the set of sequences equivalent to $\left\{\alpha x_{n}\right\}$, where $\left\{x_{n}\right\}$ is in $\hat{x}$. It is clear that these two operations are well defined, since they are independent of the choice of elements from $\hat{x}$ and $\hat{y}$. So, $\widehat{X}$ is a linear space with operations.

Theorem 13. If $X$ is a linear space having a uniformly continuous 2-norm $\|\cdot, \cdot\|$ defined on it, then for pairs of the equivalent Cauchy sequences and $\left\{x_{n}\right\} \sim\left\{a_{n}\right\}$ and $\left\{y_{n}\right\} \sim\left\{b_{n}\right\}$, then

$$
\lim _{n \rightarrow \infty}\left\|x_{n}, y_{n}\right\|=\left\|a_{n}, b_{n}\right\| .
$$

Theorem 14. If $\left\{x_{n}\right\}$ and $\left\{y_{n}\right\}$ are Cauchy sequences in a linear 2-normed space $(X,\|\cdot\|$,$) , then \left\{x_{n}-y_{n}\right\}$ is a Cauchy sequence in $X$.

Proof. We see that

$$
\begin{aligned}
\left\|\left(x_{n}-y_{n}\right)-\left(x_{m}-y_{m}\right), z\right\| & =\left\|\left(x_{n}-x_{m}\right)-\left(y_{n}-y_{m}\right), z\right\| \\
\leq K( & \left(\left\|x_{n}-x_{m}, z\right\|\right. \\
& \left.+\left\|y_{n}-y_{m}, z\right\|\right),
\end{aligned}
$$

we can readily see that when $n \rightarrow \infty,\left\{x_{n}-y_{n}\right\}$ is a Cauchy sequence in $X$.

Whenever $X$ is a space having a uniformly continuous 2-norm defined it which is possible to define real-valued function on the space $\widehat{X}$. The function is defined as follows.
For any two elements $\widehat{x}$ and $\hat{y}$ in $\widehat{X}$,

$$
\|\hat{x}, \hat{y}\|=\lim _{n \rightarrow \infty}\left\|x_{n}, y_{n}\right\|,
$$

where $\left\{x_{n}\right\} \in \hat{x}$ and $\left\{y_{n}\right\} \in \hat{y}$.

Since the limit exists and is independent of the choice of the elements in $\hat{x}$ and $\hat{y}$, the function is well defined.

Theorem 15. If $X$ is a linear space having a uniformly continuous 2-norm $\|\cdot, \cdot\|$ defined on it and $\left\{x_{n}\right\}$ and $\left\{y_{n}\right\}$ are the Cauchy sequences in $\hat{x}$ and $\hat{y}$, respectively, then the function defined by

$$
\|\widehat{x}, \hat{y}\|=\lim _{n \rightarrow \infty}\left\|x_{n}, y_{n}\right\|
$$

is a pseudo-quasi 2-norm on $\widehat{X}$.

Proof. By using definition of 2-normed spaces, we see the following:

$\left(2 N_{1}\right)$ let $\hat{x}=\alpha \hat{y}$

$$
\begin{aligned}
\|\hat{x}, \hat{y}\| & =\|\alpha \hat{y}, \hat{y}\| \\
& =\lim _{n \rightarrow \infty}\left(|\alpha|\left\|x_{n}, y_{n}\right\|\right) \\
& =0,
\end{aligned}
$$

$\left(2 N_{2}\right)$ it is easy to see as follows:

$$
\begin{aligned}
\|\hat{x}, \hat{y}\| & =\lim _{n \rightarrow \infty}\left\|x_{n}, y_{n}\right\| \\
& =\lim _{n \rightarrow \infty}\left\|y_{n}, x_{n}\right\| \\
& =\|\hat{y}, \hat{x}\|,
\end{aligned}
$$

$\left(2 N_{3}\right)$ on account of definition of 2-normed space, that is,

$$
\begin{aligned}
\|\alpha \hat{x}, \hat{y}\| & =\lim _{n \rightarrow \infty}\left\|\alpha x_{n}, y_{n}\right\|=\lim _{n \rightarrow \infty}|\alpha|\left\|x_{n}, y_{n}\right\| \\
& =|\alpha| \lim _{n \rightarrow \infty}\left\|x_{n}, y_{n}\right\| \\
& =|\alpha|\|\hat{x}, \hat{y}\|,
\end{aligned}
$$

$\left(2 N_{4}^{*}\right) \hat{x}, \hat{y}, \widehat{z} \in \widehat{X},\left\{x_{n}\right\} \in \hat{x},\left\{y_{n}\right\} \in \hat{y},\left\{z_{n}\right\} \in \widehat{z}$ are the Cauchy sequences,

$$
\begin{aligned}
\|\hat{x}, \hat{y}+\widehat{z}\| & =\lim _{n \rightarrow \infty}\left\|x_{n}, y_{n}+z_{n}\right\| \\
& \leq \lim _{n \rightarrow \infty}\left(K\left(\left\|x_{n}, y_{n}\right\|+\left\|x_{n}, z_{n}\right\|\right)\right) \\
& =K \lim _{n \rightarrow \infty}\left\|x_{n}, y_{n}\right\|+K \lim _{n \rightarrow \infty}\left\|x_{n}, z_{n}\right\| \\
& =K(\|\hat{x}, \hat{y}\|+\|\hat{x}, \widehat{z}\|) .
\end{aligned}
$$

This shows that $\|\hat{x}, \hat{y}\|=\lim _{n \rightarrow \infty}\left\|x_{n}, y_{n}\right\|$ is a pseudo-quasi2-norm on $\widehat{X}$. 
Let $\widehat{X}_{0}$ be the subset of $X$ consisting of those equivalence classes which contain a Cauchy sequence $\left\{x_{n}\right\}$ for which $x_{1}=$ $x_{2}=\cdots=x_{n}=\cdots$. At most one sequence of this kind can be in each equivalence class. If $\hat{x}$ and $\hat{y}$ are in $\widehat{X}_{0}$ and if the corresponding Cauchy sequences are $\left\{x_{n}\right\}$ and $\left\{y_{n}\right\}$ with $x_{n}=$ $x$ and $y_{n}=y$, for every $n$, then we have

$$
\|\hat{x}, \hat{y}\|=\lim _{n \rightarrow \infty}\left\|x_{n}, y_{n}\right\|=\|x, y\| .
$$

Thus, $\widehat{X}_{0}$ and $\widehat{X}$ are isometrics. This isometry will be used to show that $\widehat{X}_{0}$ is dense in $\widehat{X}$ (for details, see [3]).

Theorem 16. If $X$ is a linear space having a uniformly continuous quasi-2-norm $\|\cdot, \cdot\|$ defined on it, then $\overline{\left(\widehat{X}_{0}\right)}=\widehat{X}$ (for proof, see [3]).

Theorem 17. If $X$ is a linear space having a uniformly continuous quasi-2-norm which is defined as

$$
\|\widehat{x}, \hat{y}\|=\lim _{n \rightarrow \infty}\left\|x_{n}, y_{n}\right\|,
$$

then $\widehat{X}$ is complete and the pair $(\widehat{X},\|\cdot\|$,$) is called the$ completion of a quasi-2-normed space.

Proof. In order to see that $(\widehat{X},\|\cdot \cdot\|)$ is complete, we have to show that every Cauchy sequence in $\widehat{X}$ is convergent in $\widehat{X}$. Let $\left\{a_{n}\right\}$ be a Cauchy sequence in $\widehat{X}$ and $\widehat{b} \in \widehat{X}_{0}, \widehat{c}_{n} \in \widehat{X}_{0}$.

Because of $\overline{\left(\widehat{X}_{0}\right)}=\widehat{X}$, then we can write $\left\|\widehat{a}_{n}-\widehat{c}_{n}, \widehat{b}\right\|<1 / n$, for each $n$; also, we have,

$$
\begin{aligned}
& \left\|\widehat{c}_{n}-\widehat{c}_{m}, \hat{b}\right\|=\left\|\widehat{c}_{n}-\widehat{a}_{n}+\widehat{a}_{n}-\widehat{c}_{m}, \hat{b}\right\| \\
& \leq K\left(\left\|\widehat{c}_{n}-\widehat{a}_{n}, \widehat{b}\right\|+\left\|\widehat{a}_{n}-\widehat{c}_{m}, \hat{b}\right\|\right) \\
& =K\left(\left\|\widehat{c}_{n}-\widehat{a}_{n}, \hat{b}\right\|+\left\|\widehat{a}_{n}-\widehat{a}_{m}+\widehat{a}_{m}-\widehat{c}_{m}, \widehat{b}\right\|\right) \\
& \leq K\left\|\widehat{a}_{n}-\widehat{c}_{n}, \hat{b}\right\| \\
& +K\left(K\left\|\widehat{a}_{n}-\widehat{a}_{m}, \widehat{b}\right\|+\left\|\widehat{a}_{m}-\widehat{c}_{m}, \hat{b}\right\|\right), \\
& \text { for } K \geq 1 \\
& <\frac{K}{n}+K^{2}\left\|\widehat{a}_{n}-\widehat{a}_{m}, \widehat{b}\right\|+\frac{K^{2}}{m} .
\end{aligned}
$$

Last from inequality when $n, m \rightarrow \infty$ right hand side will be equal to 0 . Thus

$$
\lim _{n, m \rightarrow \infty}\left\|\widehat{c}_{n}-\widehat{c}_{m}, \widehat{b}\right\|=0
$$

this shows us that $\left\{\widehat{c}_{n}\right\}$ is a Cauchy sequence in $\widehat{X}$.

Use of $\widehat{X}$ and $\widehat{X}_{0}$ are isometric there is a Cauchy sequence $\left\{c_{n}\right\}$ in $X$ that corresponding $\left\{\widehat{c}_{n}\right\}$.
On the other hand, there is $\widehat{a} \in \widehat{X}$ such that $\widehat{a} \in\left\{\widehat{c}_{n}\right\}$

$$
\begin{aligned}
\left\|\widehat{a}_{n}-\widehat{a}, \hat{b}\right\| & =\left\|\widehat{a}_{n}-\widehat{c}_{n}+\widehat{c}_{n}-\widehat{a}, \hat{b}\right\| \\
& \leq K\left(\left\|\widehat{a}_{n}-\widehat{c}_{n}, \widehat{b}\right\|+\left\|\widehat{c}_{n}-\widehat{a}, \widehat{b}\right\|\right) \\
& <\frac{K}{n}+K\left\|\widehat{c}_{n}-\widehat{a}, \hat{b}\right\| .
\end{aligned}
$$

Last from the inequality as $n \rightarrow \infty$ and $\widehat{X}_{0}$ is dense in $\widehat{X}$,

$$
\lim _{n \rightarrow \infty}\left\|\widehat{a}_{n}-\widehat{a}, \widehat{b}\right\|=0 .
$$

So, arbitrary of a Cauchy sequence $\left\{\widehat{a}_{n}\right\}$ is convergent to $\widehat{a} \epsilon$ $\widehat{X}$. Then, $(\widehat{X},\|\cdot, \cdot\|)$ is complete.

\section{Acknowledgment}

The authors would like the thank to referees for their suggestions and comments, which have improved the presentation of the paper.

\section{References}

[1] K. Menger, "Untersuchungen Veber allgeine Metrik," Mathematische Annalen, vol. 100, 1928.

[2] S. Gähler, "Lineare 2-normierte räume," Diese Nachr, vol. 28, no. 1-2, pp. 1-43, 1965.

[3] R. W. Freese and Y. J. Cho, Geometry of Linear 2-Normed Spaces, Nova Science, Hauppauge, NY, USA, 2001.

[4] C. Park, "Generalized quasi-Banach spaces and quasi -(2, p) normed spaces," Journal of the Chungcheong Matematical Society, vol. 19, no. 2, 2006.

[5] A. G. White Jr., 2-Banach Spaces, St. Bonaventure, New York, NY, USA, 1967. 


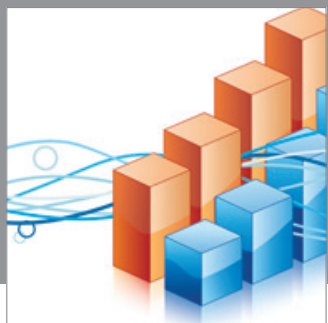

Advances in

Operations Research

mansans

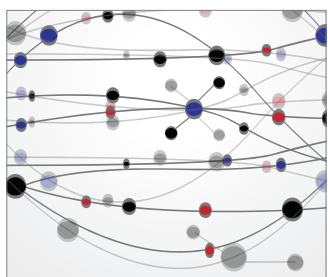

The Scientific World Journal
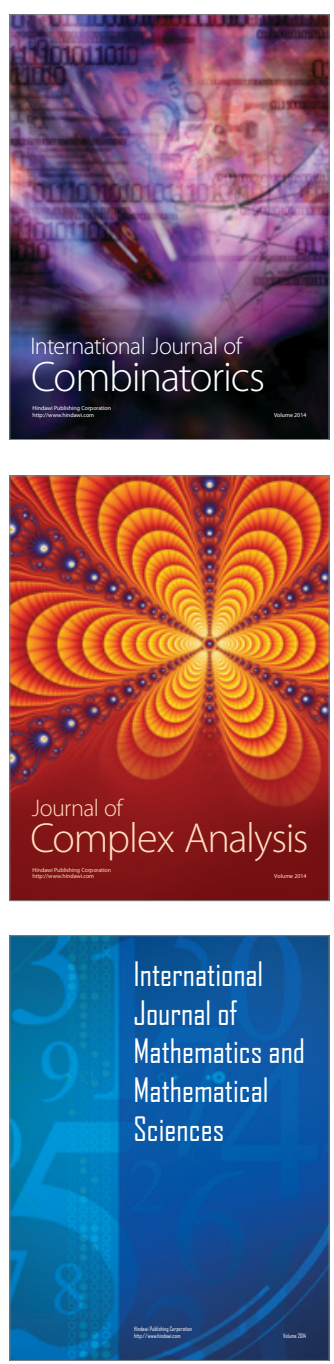
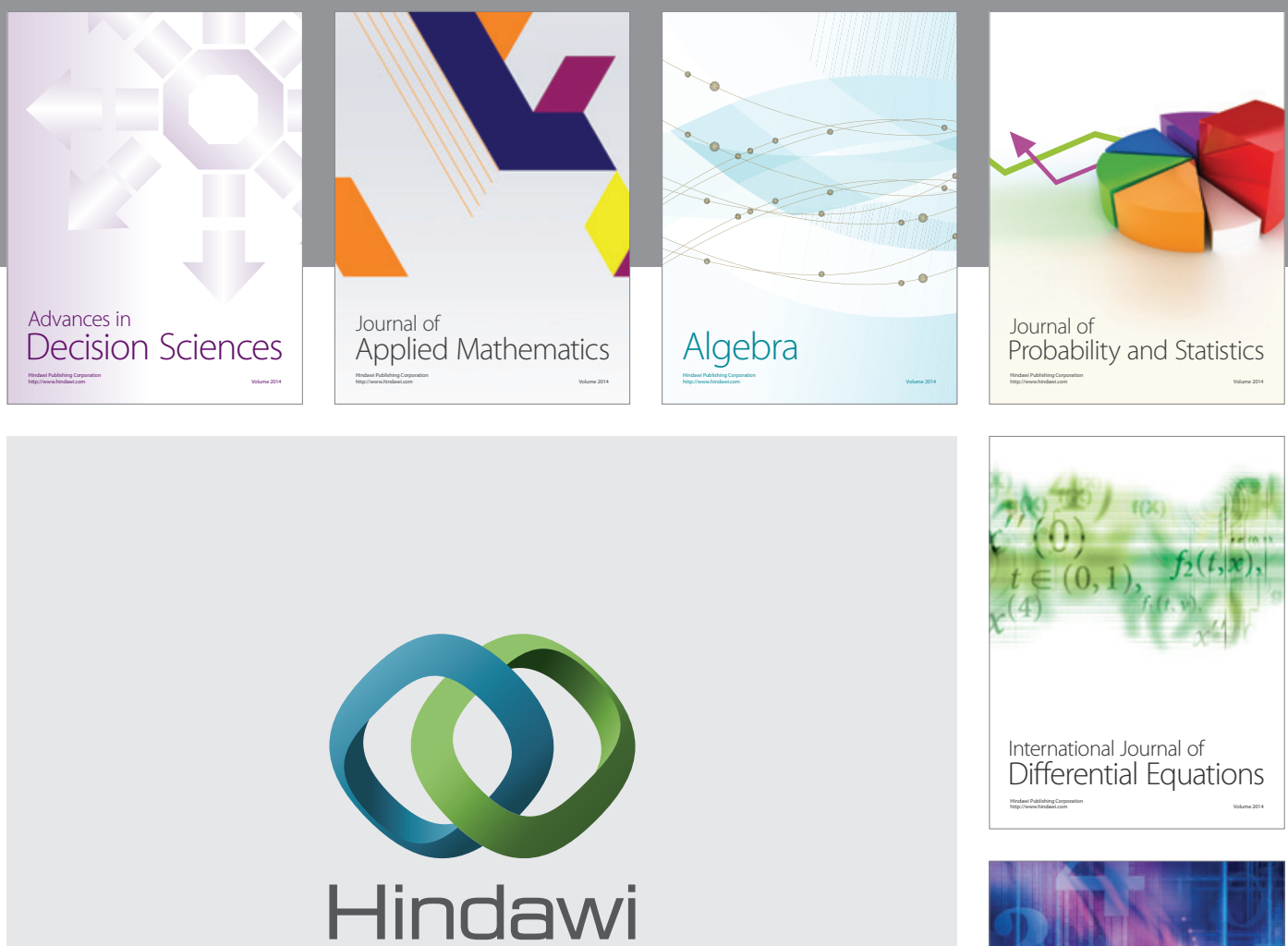

Submit your manuscripts at http://www.hindawi.com
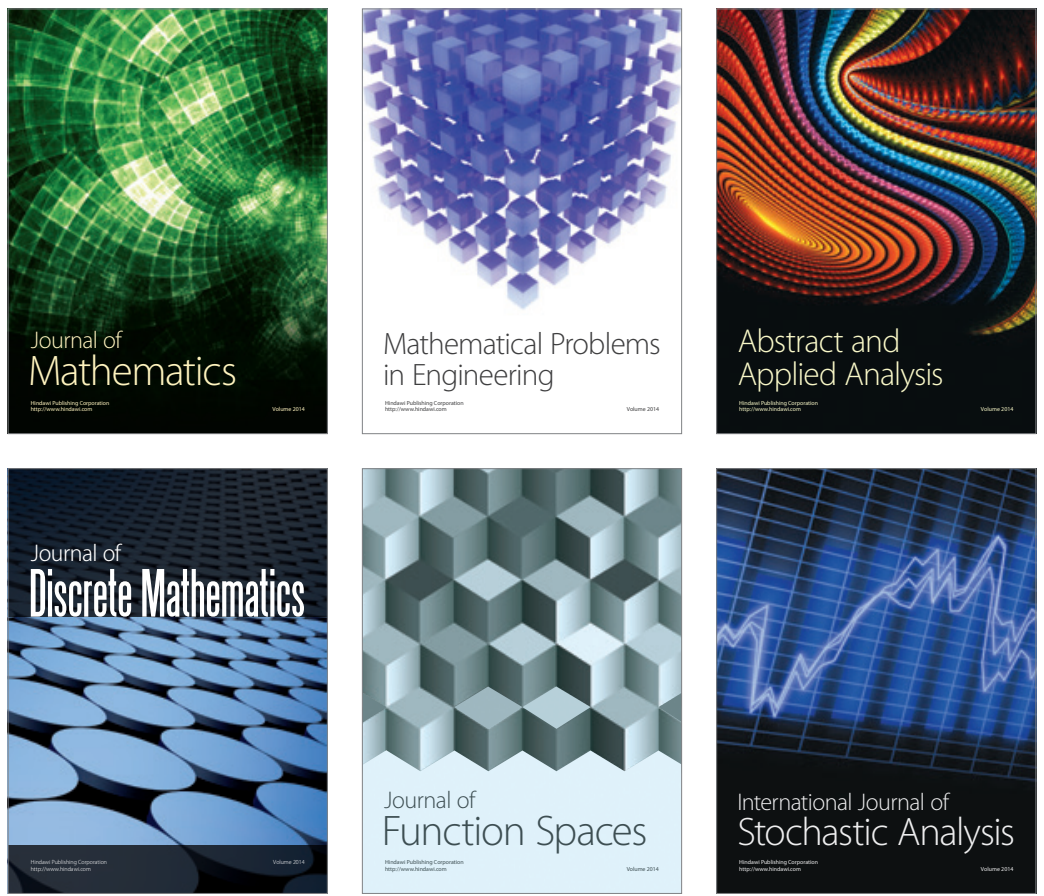

Journal of

Function Spaces

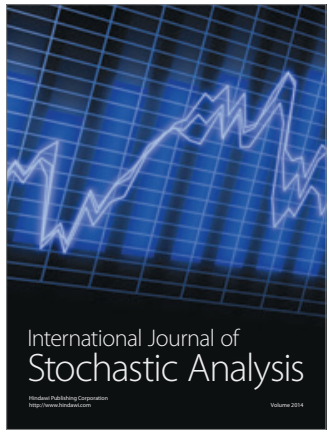

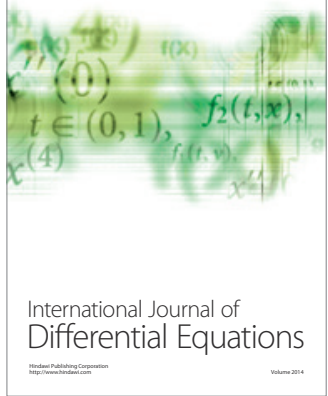
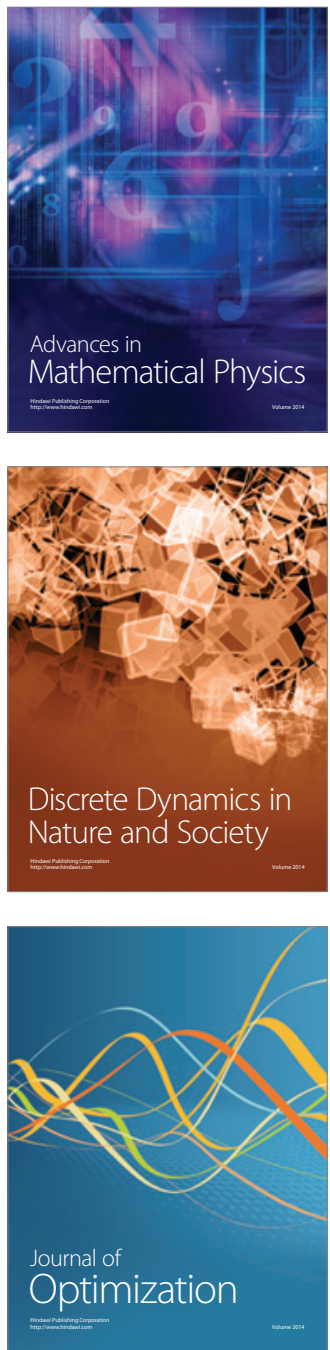